\title{
Residualidad de plaguicidas en suelos dedicados al cultivo de banano dominico (musa spp.) en Tlapacoyan Veracruz y sus posibles efectos a la salud
}

\author{
Alan Guzmán Cabrera1,2 - Juan Carlos Noa Carrazana² - María Ariadna Escalante \\ Rebolledo³ - Paloma Violeta Susan Tepetlán²
}

\begin{abstract}
RESUMEN
El uso irracional de plaguicidas, para el control de plagas y enfermedades, altera las propiedades naturales del suelo y lo contamina, así como causar daños a la salud. Al superar la capacidad de amortiguamiento del suelo, repercute en sus propiedades físicas, químicas y biológicas,

los cuales influyen en la movilidad y degradación de estos compuestos. El objetivo de este trabajo fue evaluar la residualidad de los plaguicidas en suelos dedicados a la producción de banano dominico (Musa spp.) en el municipio de Tlapacoyan, Veracruz y sus posibles efectos que podrían causar a la salud humana por una constante exposición o consumo de alimentos que contengan residuos de plaguicidas. Los resultados de los análisis de caracterización edáfica

y multi-residual de altos insumos, detectaron un alto contenido de materia orgánica, lo cual favorece la retención de compuestos de plaguicidas con características lipófilas, se encontró un índice de 0.004 (mg.kg-1) de la molécula 1,4 DDE (Organoclorado) un derivado del DDT y no se encontraron residuos de otros plaguicidas. Sin embargo, es necesario evaluar la residualidad de los plaguicidas con metodologías más específicas. En cuanto a los problemas de salud, no hay reporte de enfermedades crónicas, pero se sabe que los plaguicidas han intoxicado a trabajadores del campo provocando vómito, lesiones dermatológicas y asfixia por inflamación de lengua

(Glositis) entre otras afectaciones.
\end{abstract}

\begin{abstract}
The irrational use of pesticides, for the control of pests and diseases, pollutes the environment and causes damage to the health, by overcoming the soil's buffering capacity, has repercussions on its physical, chemical and biological properties, which influence the mobility and Degradation of these compounds. The objective of this work was to evaluate the residuality of pesticides in soils dedicated to the production of Dominican bananas (Musa paradisiaca) in Tlapacoyan, Ver. And the possible health effects caused by the exposure or ingestion in relation to pesticides used in the soil. The results of the multiresidues of high input analyzes only detected an index of 0.004 (mg.kg-1) of the molecule 1,4 DDE (Organochlorine) a derivative of DDT and no residues of other pesticides were found. However, it is necessary to evaluate pesticides with other more specific methodologies.
\end{abstract}

\footnotetext{
1 Facultad de Ciencias Químicas, Ing. Ambiental, Xalapa Circuito Gonzalo Aguirre Beltrán s/n Zona Universitaria C.P. 91090 Xalapa, Veracruz, Méx.

2 Instituto de Biotecnología y Ecología Aplicada, Universidad Veracruzana, Av. de las Culturas Veracruzanas No. 101, Campus para la Cultura, las Artes y el Deporte, Col. Emiliano Zapata, C.P. 91090, Xalapa, Veracruz, Méx.

3 Facultad de Ciencias Agrícolas, Xalapa Circuito Gonzalo Aguirre Beltrán s/n Zona Universitaria C.P. 91090 Xalapa, Veracruz, Méx.

gc.alan28@gmail.com,jnoa@uv.mx, arilel@hotmail.com,palomasusant@gmail.com.
} 
Introducción

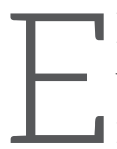

l campo agrícola, se enfrenta a distintas adversidades, el latente cambio climático ha impactado con intensas lluvias, fuertes vientos y sequías extremas. Una de las problemáticas que debería de acaparar una mayor relevancia, es la producción bajo la implementación de insumos agroquímicos (fertilizantes y plaguicidas). Los cuales cuando son empleados de una manera irracional contribuye principalmente a la acidificación y salinización de suelos. En particular el uso de plaguicidas para el control de plagas y enfermedades, pone en riesgo la salud, daña al ambiente y eleva los costos de producción (Echarri, 2016; García, 2017). Sin embargo, con el fin de asegurar la producción y adaptarse a la creciente demanda alimenticia ha crecido la demanda de estos insumos.

Los plaguicidas empleados son sustancias ajenas al sistema, contaminan e interfieren con los procesos biológicos del suelo aun cuando sean efectivos en el control de las plagas, al ser liberados al ambiente sufren transformaciones por distintos procesos, lo cual puede dar como resultado una degradación del componente o un metabolito más persistente, los cuales pueden ser adsorbidos por las partículas del suelo y permanecer con efectos residuales o lixiviar a mantos freáticos. Además, pueden volatilizarse y recorrer grandes distancias hasta caer por acción de la gravedad en forma seca o mezclarse con partículas de agua y depositarse en forma de lluvia, esto agrava el problema de contaminación de plaguicidas, por su movilidad a otros medios (Lagrega, 1996).

La residualidad de los plaguicidas en el ambiente, tienden a tener propiedades lipófilas, los cuales favorecen la adsorción y acumulación de estos compuestos en aceites vegetales o ceras, por tal razón, podría presentarse concentraciones de compuestos de plaguicidas en cáscaras de frutos y tanto fauna silvestre como humanos, pueden exponerse a estos compuestos, por la ingesta de alimentos contaminados con plaguicidas (Hayes y Laws, 1991). Posiblemente los plaguicidas puedan ser extraídos del suelo y traslocarse a través de la raíz de la planta hasta llegar al fruto y bioacumularse (Gimenez, 1991).

En el Estado de Veracruz, se cultivan al menos 10 variedades de plátanos desde cultivos puros tecnificados, hasta parcelas a nivel de traspatio con alto grado de diversificación agrícola, de las cuales el plátano dominico es una de las más cultivadas. La municipalidad de Tlapacoyan destaca en la siembra y cosecha de variedades de plátanos como: Dominicos, Morados, Largos, entre otros, imprimiendo esta actividad un fuerte carácter de dependencia económica a la población de la región (Noa-Carrazana y Flores-Estévez, 2008). El cultivo de banano dominico se posiciona en segundo lugar entre los productos de alto valor productivo con un promedio de 5,838.6 millones en el período 2013 (FND, 2014; SAGARPA, 2005). Para qué el comercio de banano dominico alcance el mercado internacional, debe cumplir con los estándares de inocuidad de países qué se desea exportar este producto. Estos estándares, incluyen todas las acciones qué garantizan la máxima seguridad de los alimentos, lo cuales no deben contener agentes físicos, químicos (plaguicidas) y biológicos, que pongan en riesgo la salud. La exposición e ingesta de plaguicidas puede ocasionar problemas crónicos de salud. Los humanos compartimos funciones fisiológicas y bioquímicas con organismos que dañan los cultivos, los cuales buscan ser erradicados con el uso de plaguicidas, sin embargo, se emplean millones de kilogramos al año de estos compuestos y a causa de su alta movilidad y persistencia en el ambiente, podríamos estar expuestos sin tener conciencia de ello (alimentos, agua, inhalación y exposición) y con el paso del tiempo padecer alguna enfermedad causada por la bioacumulación de los plaguicidas en nuestros cuerpos (Karam et al., 2004; OMS, 2016). Desafortunadamente en México se emplean alrededor de 186 plaguicidas altamente nocivos prohibidos en países primermundistas y otros prohibidos en México, los cuales son comercializados y empleados clandestinamente (Fregoso, 2014). 
Materiales y métodos

El trabajo experimental se dividió en tres fases; de campo, donde se realizaron encuestas a tiendas agroquímicas, productores de banano dominico y muestreo de suelo con diferente tipo de manejo. De laboratorio, donde se realizaron análisis para la caracterización edáfica y determinación de compuestos residuales de plaguicidas. Y consulta bibliográfica sobre problemas de salud causados por el uso y exposición de plaguicidas, análisis y discusión de resultados.

Tlapacoyan Veracruz, se localiza en la zona centro del estado (figura 1), en las coordenadas $19^{\circ} 58^{\prime}$ latitud norte y $97^{\circ} 13^{\prime}$ longitud oeste a una altura de 430 metros sobre el nivel del mar. Limita al noroeste con Martínez de la Torre, al sureste con Atzalan, al Sur con Jalacingo. Cuenta con una superficie total de 167.97 $\mathrm{km} 2$.

Su clima es cálido-húmedo-regular con temperatura promedio de $18^{\circ}$. Su vegetación es de tipo bosque subtropical perennifolio. Su suelo es de tipo luvisol, con contenido de arcilla, sus- ceptible a la erosión, y se utiliza principalmente para la agricultura y ganadería (Herrera et al., 2017).

Encuestas realizadas a tiendas agroquímicas del municipio de Tlapacoyan, Veracruz.

Por la disponibilidad de las tiendas agroquímicas se encuestaron a 5, para recabar información que nos ayude a identificar los plaguicidas disponibles para los cultivos de banano dominico y validar con los productores sí coinciden con los empleados en sus parcelas. Se buscó conocer si ofrecen asesorías a productores sobre su manejo.

Encuesta empleada a los productores de banano dominico

La encuesta se aplicó a 50 productores elegidos de forma aleatoria, para determinar el tipo de manejo en el control de plagas y enfermedades, frecuencia, dosificación en las diferentes parcelas y su conocimiento acerca del riesgo de

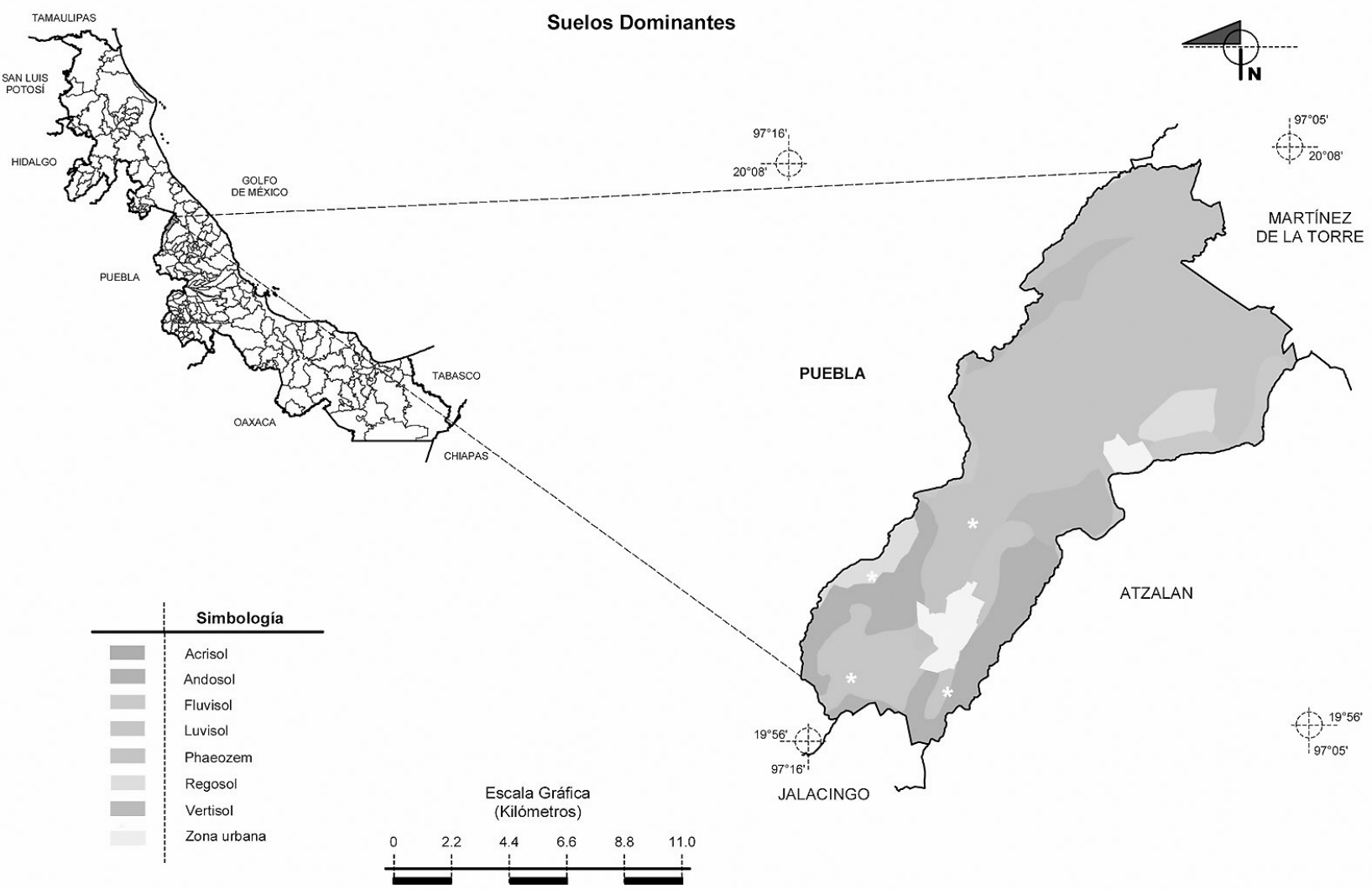

Figura 1. Localización de los sitios en estudio del municipio de Tlapacoyan Veracruz (Fuente: INEGI, 2009). 
implementar estas sustancias en sus cultivos, así como la capacitación que hayan recibido al respecto.

\section{Muestreo de suelos}

Con base a las encuestas y a la accesibilidad de los terrenos, se eligieron 6 parcelas con 3 tipos de manejo; 2 con frecuencia de manejo durante todo el año dentro de un rango de 15 días (altos insumos), 2 de 30 a 60 días (bajos insumos) y 2 sin uso de plaguicidas u otros agroquímicos (manejo orgánico) (Tabla 1).

Independientemente de la superficie y forma de las parcelas, se tomó una hectárea en el centro de cada una de ellas, donde se colectaron 5 submuestras de $1 \mathrm{Kg}$ cada una, con el fin de obtener una muestra compuesta representativa del manejo y evitar la influencia de las parcelas colindantes.

Caracterización edáfica y Análisis multi-residual de plaguicidas

En el Laboratorio de Suelos de la facultad de Ciencias Agrícolas, de la Universidad Veracruzana, Campus Xalapa, se realizó el análisis de las características físicas y químicas del suelo para cada parcela muestreada, de acuerdo con el manual fertilidad y nutrición de Escalante y May, 2008 y se correlacionó las propiedades físicas y químicas del suelo, con la residualidad de los plaguicidas. Un laboratorio nacional privado se encargó de realizar el análisis Multi-Residual de plaguicidas por cromatografía de gases por el método de AOAC 17th Ed. Official Method 970.52 M. Organochlorine and Organophosphorus Pesticide Residues, el cual permitió conocer los componentes residuales presentes en el suelo.

\section{Resultados}

Datos obtenidos de las tiendas agroquímicas y la efectividad de las asesorías en productores

Los principales plaguicidas comercializados son: insecticida aceite parafínico (Citrolina), insecticida carbofurano (Furadán), fungicida piraclostrobina (legasus), fungicida mancozeb (Mnzate), fungicida propiconazol (Sanazole), fungicida pyrimetanil (Siganex), herbicida glifosato (Faena) y herbicida paraquat (DiabloQuat). Nuestros resultados revelan que una gran cantidad de los pesticidas utilizados en el

\begin{tabular}{|c|c|c|c|c|c|}
\hline $\begin{array}{l}\text { Clave de } \\
\text { Muestra }\end{array}$ & Ubicación & $\begin{array}{l}\text { Tipo } \\
\text { de }\end{array}$ & $\begin{array}{l}\text { Plaguicidas } \\
\text { empleados }\end{array}$ & $\begin{array}{c}\text { Frecuencia } \\
\text { de aplicación }\end{array}$ & $\begin{array}{l}\text { Tiempo } \\
\text { de uso }\end{array}$ \\
\hline 1 & $\begin{array}{l}\text { Santiago } \\
\text { Segundo }\end{array}$ & Bajos insumos & $\begin{array}{l}\text { Citrolína } \\
\text { Sanazole } \\
\text { Manzate }\end{array}$ & 30 a 45 días & 2 años \\
\hline 2 & $\begin{array}{l}\text { Santiago } \\
\text { Segundo }\end{array}$ & Bajos insumos & $\begin{array}{c}\text { Citrolina } \\
\text { Sanazole } \\
\text { Faena }\end{array}$ & 30 días & 2 años \\
\hline 3 & Rancho Nuevo & $\begin{array}{c}\text { Altos } \\
\text { insumos }\end{array}$ & $\begin{array}{l}\text { Sanazole } \\
\text { Faena } \\
\text { Diabloquat }\end{array}$ & 30 días & 6 años \\
\hline 4 & Rancho Nuevo & Altos & $\begin{array}{l}\text { Sanazole } \\
\text { Faena } \\
\text { Diabloquat }\end{array}$ & 30 días & 10 años \\
\hline \multirow[t]{2}{*}{5} & Platanozapan & Orgánico & N.A & N.A & N.A \\
\hline & & Orgánico & N.A & N.A & N.A \\
\hline
\end{tabular}

N/A: No aplica 
manejo de las parcelas (Tabla 1) tienen como fin el combate de enfermedades de las hojas del plátano, entre ellas la Sigatoka negra (Mycosphaerella fijiensis Morelet), siendo la más distribuida y agresiva para el cultivo en la región de Tlapacoyan (Aguilar-Lara et. al., 2011). Un 80\% de las tiendas agroquímicas mencionaron que proporcionaban asesoría técnica para el manejo de plaguicidas (Montoya, 2014), aunque no definieron si era de una forma integral. Todas ellas cuentan con personal con estudios superiores de diferentes ramas, sin embargo, no todos están especializados en el uso de plaguicidas. En cuanto al uso de plaguicidas, las sobredosis y las constantes aplicaciones, evidencia el contraste con las buenas prácticas agrícolas y un conocimiento empírico adquirido por muchos productores (Milton et al., 2009). Los Productores al no seguir un protocolo de manejo y disposición de dichos envases, resulta en una disposición de estos sobre las parcelas tanto de altos y bajos insumos, incineración inadecuada o en algunos casos su reutilización. Los productores emplean pañuelos o cubrebocas como protección personal al momento de aplicar los plaguicidas, esto incrementa el riesgo de intoxicación (Pereira et al., 2011; SAGARPA, 2012).

Manejo de los plaguicidas empleado por los productores en los cultivos de banano dominico

Los plaguicidas empleados por los productores coinciden con los datos recabados en las encuestas realizadas a tiendas agroquímicas, sin embargo, existe una problemática en el uso de estos productos, debido a que 60\% de los encuestados elevaron las dosis para asegurar su efectividad. El uso de plaguicidas más severos, sobredosis y mezcla de plaguicidas a causa de la resistencia generada por las plagas, superan los procesos de depuración del suelo, lo cual provoca una acumulación y residualidad de compuestos tóxicos (Porta et al., 1999; Uzcátegui et al., 2011).

Los datos de relevancia obtenidos en la encuesta son los siguientes:
- Un 90 \% de los productores encuestados emplean plaguicidas.

- La frecuencia de uso durante todo el año se encuentra dentro de un rango de 15 días (altos insumos), 30 a 60 días (bajos insumos).

- Un 90\% de los productores han recibido asesoría para el manejo de plaguicidas.

- Un 30\% de los productores usan protección mínima como lo son cubrebocas o pañuelos para el manejo de plaguicidas.

- Los casos de intoxicación por manipulación de plaguicidas han sido cuatro, qué van desde lesiones dérmicas, glositis y vómito.

- Un 30 \% de los productores optan por guardar los plaguicidas nuevos dentro del hogar, esto representa un peligro por posibles derrames o manipulación por infantes.

- Un 60\% de los encuestados elevan las dosis para contrarrestar la resistencia de las plagas.

- Los envases de los plaguicidas, son incinerados, tirados en las parcelas, enterrados, reutilizados o depositados a la basura común.

Estos resultados definen la problemática en las prácticas agrícolas, en especial, el uso inadecuado de plaguicidas, un factor importante en la residualidad de estos en el suelo (Montoya, 2014). Es importante resaltar qué las etiquetas de los plaguicidas hacen mención de los efectos a corto plazo similares a este tipo de efectos, sin embargo, no citan los problemas de salud qué pueden ocasionar a largo plazo (Fregoso, 2014).

\section{Resultados de la caracterización edáfica}

Los parámetros evaluados en los suelos muestran que en todas las parcelas existen $\mathrm{pH}$ que van de moderada a fuertemente ácidos, con valores dentro de un rango de 5.4 a 4.8. El contenido de materia orgánica según la norma oficial (NOM-021-SEMARNAT-2000), los considera como medios o bajos, sin embargo, otros estudios los consideran como adecuados o ri- 
cos, corroborándose en la densidad aparente, como en el caso de la parcela ubicada en Rancho Nuevo y Platanozapan qué presentaron valores de 6.14 y 3.70 en Rancho Nuevo , 9.85 Platanozapan (Aguilar et al., 1987; Andrade y Martínez, 2014; DOF, 2000). La densidad aparente es baja, con valores de .78 g.cm-3 y .90 g.cm-3 en Rancho Nuevo, .75 g.cm-3 en Platanozapan, para las texturas presentadas que van de arcillas a migajones arcillosos, que podemos pensar que es por el contenido de materia orgánica (Narro, 1994). En cuanto a los contenidos nutricionales todos son ricos de $\mathrm{Ni}$ trógeno y presentan rangos de Fósforo que van de medios a bajos (Andrade y Martínez, 2014). En cuanto a las bases cambiables, el Potasio se encuentra muy bajo, cuando el requerimiento de este nutriente para el cultivo de banano es alto (Furcal y Barquero, 2014); el Calcio desde adecuado a bajos, magnesio alto y Sodio de adecuados a ligeramente altos, este desbalance vuelve a las plantas más susceptibles al ataque de plagas y enfermedades (Agrios, 2010; Ministerio de Agricultura, 1994).

Resultado de los análisis multi-residual por cromatografía de gases

El análisis multi-residual determinó un contenido de 0.004 mg.kg-1 DDE en la parcela ubicada en Rancho Nuevo, su valor es mucho menor a los LMRP de normas Internacionales de la Agencia de Protección Ambiental (USEPA) de los EUA (Bournicore, 1996), ya que en México no existe una norma que regule la contaminación del suelo provocada por plaguicidas. La presencia de este metabolito se debe al alto grado de acidez del suelo, para fitosanitarios con posibilidad de protonación, que elevaría la adsorción con las arcillas del suelo; por otra parte, el contenido de materia orgánica de esta muestra fue mayor comparada a las demás de manejo con plaguicidas, lo cual por procesos de adsorción y degradación disminuyen la toxicidad del plaguicida e incrementa su vida media (Porta et al., 1999). El resto de los plaguicidas fue perdido, probablemente por lixiviación e incluso degradados por microrganismos anae- robios que incrementan su actividad en suelos inundados y ricos en materia orgánica, ya que el periodo entre la aplicación de los productos y el muestreo presentó condiciones climáticas de muy altas precipitaciones, aunado a la alta solubilidad de los productos (Fernández y Dorronsoro, 2016; Sparks, 1995; Castro y Yoshida, 1971).

Los plaguicidas se han convertido en la forma dominante para el control de plagas y enfermedades, sin embargo, el desconocimiento de la población acerca de sus repercusiones al ambiente y salud humana, ha contribuido a un aumento en el uso de estos compuestos. Los principales afectados son los productores del campo, muchos de ellos no usan el equipo apropiado para su protección, lo cual incrementa el riesgo de intoxicación.

Los insecticidas organoclorados inhiben la comunicación intercelular y actúan como promotores de tumores, en algunos casos los expuestos han padecido cáncer de pulmón, de vejiga, cerebro y los sistemas linfáticos y hematopoyético (Karam et al., 2004).

Una intoxicación por vía oral del Dicloro Difenil Tricloroetano (DDT), puede provocar lo siguiente:

Hiperestesias en boca y parte inferior de la cara, seguida de parestesias, confusión, malestar, cefalea y fatiga, acompañado de vómitos, dolor abdominal y diarrea. En caso de intoxicaciones graves se producen convulsiones con pérdida de conciencia.

En los últimos años, el uso de herbicidas ha aumentado, puesto que resulta más económico acabar con la maleza con compuestos químicos qué de forma manual, con respecto a ellos, los daños provocados por la intoxicación aguda por el ingrediente activo Paraquat tiene predilección por tejidos con saturación importante de oxígeno como el pulmón, hígado y riñon, mientras que en casos de intoxicación crónica se han relacionado con Parkinsonismo, cabe destacar que el uso del Paraquat está restringido en México, sin embargo, es uno de los herbicidas más empleados. Recientemente el Glifosato ha adquirido fama por su efectividad en el control de malezas y su extensa comercialización, sin embargo, se ha relacionado con 
posibles efectos citotóxicos y genotóxicos y se ha clasificado como posible carcinógeno para los seres humanos por la Agencia Internacional de Investigación del Cáncer de la Organización Mundial de la Salud (Bernardino et al., 2016; Ferrer, 2003).

La problemática no sólo invade al campo, las personas que ingieren alimentos contaminados con plaguicidas son susceptibles a una bioacumulación de estos compuestos en su organismo. La leche materna es uno de los principales indicadores de la contaminación ambiental a la que es sometida la población, ya que la lactancia supone una continua movilización de estas sustancias desde el tejido adiposo a la leche. Se ha demostrado que el feto puede sufrir mutaciones y es vulnerable a la contaminación por biocidas organoclorados durante la gestación, esto se asocia a un inadecuado desarrollo, enfermedades en la niñez y adultez, situaciones que en definitiva afectan la calidad de vida del niño (Karam et al., 2004; Per, 2008).

\section{Conclusiones}

Los plaguicidas de acuerdo a su composición química de mayor uso en la región Tlapacoyan Ver. son: insecticida aceite parafínico (Citrolina), insecticida carbofurano (Furadán), fungicida piraclostrobina (legasus), fungicida mancozeb (Mnzate), fungicida propiconazol (Sanazole), fungicida pyrimetanil (Siganex), herbicida glifosato (Faena) y herbicida paraquat (DiabloQuat). Con respecto a la residualidad de los plaguicidas empleados, sólo se presentó en la parcela de altos insumos (muestra 3) ubicada en Rancho Nuevo, el metabolito Dicloro Difenil Tricloro Etileno a una concentración de 0.004 mg.kg-1; un compuesto resultado de la degradación y uso en el pasado del Dicloro Difenil Tricloroetano (Organoclorado), actualmente se encuentra prohibida su comercialización, aunque en algunos lugares del país se adquiere de manera clandestina, éste plaguicida se absorbe por la piel, aparato respiratorio y digestivo, son raros los casos de intoxicación aguda por este plaguicida, sin embargo; por su alta solubilidad en grasas se acumula en tejidos grasos, incluyendo la leche materna; los problemas de salud que se pueden presentar por el uso de Organoclorados son: cáncer de vejiga, aparato reproductor, cerebro y de sistemas linfáticos y hematopoyético, son promotores de tumores, algunos casos de cáncer de pulmón, de igual manera interferir en la cadena trófica o afectar a la procreación y lactancia (Karam et al., 2004;Der, 2008). Las 4 personas intoxicadas por insecticidas son productores y la intoxicación se atribuye a exposición durante la aplicación de estos.

Las características físicas y químicas de los suelos estudiados registran una baja fertilidad en todos ellos comparándolos con la normativa NOM-021-SEMARNAT-2000, sin embargo, los criterios de Aguilar et al., (1987), son considerados como ricos.

No existieron residuos de plaguicidas en la mayoría de las parcelas debido a que características físicas y químicas de los suelos como $\mathrm{pH}$, contenido de materia orgánica, porcentaje de arcilla, probablemente hayan metabolizado o retenido tales productos. El alto contenido de materia orgánica en particular asegura una gran biomasa microbiana que influirá en la degradación o retención de los plaguicidas.

En la Zona de Tlapacoyan, los productores emplean plaguicidas para el control de plagas y enfermedades, sin embargo, no aplican las buenas prácticas agrícolas, esto pone en riesgo la salud de las personas que frecuenten estos productos, y por su posible movilidad a las personas que habiten el sitio, por esta razón es de suma importancia que personas especializadas en el uso de plaguicidas impartan conferencias sobre los riesgos que representan, tanto a la salud cómo al medio ambiente.

\section{Referencias}

Agrios George. (2010). Efecto del ambiente en la producción de las enfermedades infecciosas. En Fitopatología(p154). México: Limusa.

Aguilar A., Etchevers, J. D., y Castellanos , J. Z. (1987). Análisis químico para evaluar la fertilidad del suelo. México: Sociedad Mexicana de la Ciencia del Suelo.

Aguilar-Lara, J.L., Noa-Carrazana J.C., Córdova-Nieto, 
C., y Ortiz-Ceballos, G.C. (2011). Presencia de sigatokas (Mycospharella sp) en cultivares diversificados de plátanos, en el municipio de Tlapacoyan, Veracruz. En: Memorias XXIV Reunión Científica-Tecnológica Forestal y Agropecuaria Veracruz y III Del Trópico Mexicano. Edit. INIFAP-CIR Golfo. Centro. pp.97-100.

Andrades, M., y Martínez, E. (2014). Fertilidad del suelo y parámetros que la definen. Agricultura y Alimentación. $3^{\circ}$ Edición. pp. 19.

Bernardino H., Mariaca R., Nazar A., Álvarez J., Torres A. y Herrera C. (2016). Exposición a plaguicidas en campesinos indígenas en los Altos de Chiapas, México. Revista Mexicana de Agroecosistemas, 3, 356.

Bournicore, A. (1996). Cleanup criteria for contaminated soil and ground-water. EUA: Astm International.

Diario Oficial de la Federación. (2000). NOM-021-RECNAT-2000. SEMARNAT

Der S. (2008). Plaguicidas organoclorados en leche materna. Revista del Hospital Materno Infantil Ramón Sardá, 27, 70-78 pp.

Echarri, L. (09 de Noviembre de 2016). Impactos ambientales de la agricultura moderna. Obtenido de Ciencias de la tierra y del medio ambiente: http://www4.tecnun.es/ asignaturas/Ecologia/Hipertexto/06Recursos/121ImpactAmbAgr.htm\#POBLACION

Escalante, A., y May, T. (2008). Manual de fertilidad y nutrición. México: Facultad de Ciencias Agrícolas de la Universidad Veracruzana.

Fernández y Dorronsoro Carlos. (27 de Agosto de 2016). Contaminación por fitosanitarios. Plaguicidas. Recuperado, Septiembre de 2016, de Libros web, monografías, trabajos de investigación, conferencias, atlas sobre la Edafología y los suelos : http://www.edafologia.net/conta/ tema13/evol.htm

Ferrer A. (2003). Intoxicación por plaguicidas. 02 de Junio del 2017, de SCIELO Sitio web: http:// scielo.isciii.es/scielo.php?script=sci_arttext $\&$ pi$\mathrm{d}=$ S1137-66272003000200009

Financiera Nacional de Desarrollo. (2014). Panorama de plátano. Dirección General Adjunta de Planeación Estratégica, Análisis Sectorial y Tecnologías de la Información. México.

Fregoso, J. (2014). En México se usan 186 plaguicidas altamente peligrosos, denuncian activistas; algunos fueron prohibidos en Europa. Sin embargo, 1.

Furcal, P., y Barquero, A. (2014). Fertilización del plátano con nitrógeno y potasio durante el primer ciclo productivo. México: Agronomía mesoamericana. 25-26.
García Lorenzo y García Carlos. (23 de marzo del 2017). Adversidades Agroclimáticas. Ministerio de Agricultura, Pesca y Alimentación, 20/82, 3-12.

Gimenez, I. (1991). Ensayos sobre la translocación en plantas de fungicidas sistémicos. Boletín de sanidad vegetal: Plagas. pp. 497-518.

Hayes, J., Wayland, J., Laws, R., y Edward, J. (1991). Effects of pesticides on wildlife. San Diego, California: Academic Press, Inc. pp. 465-466.

Herrera F., Escobar R., y Benítez A. (2017). Tlapacoyan. 2017, de Enciclopedia de los municipios y estados de México Sitio web: http://siglo.inafed.gob.mx/enciclopedia/ EMM30veracruz/municipios/30183a.html

INEGI. 2009. Prontuario de información geográfica municipal de los Estados Unidos Mexicanos. Tlapacoyan, Veracruz de Ignacio de la Llave. Clave geoestadística 30183. Sitio Web. http://www3.inegi.org.mx/contenidos/app/mexicocifras/datos_geograficos/30/30023.pdf

Lagrega Michael D, P. L. (1996). Gestión de residuos tóxicos, tratamiento, eliminación y recuperación de suelos. Madrid: McGrawhill.

Karam Miguel, Ramírez, Montes Patricia, Galván Juan. (3 de noviembre del 2004). Plaguicidas y salud de la población. Red de Revistas Científicas de América Latina, el Caribe, España y Portugal Sistema de Información Científica, 11-3, 246-254.

Milton Jorge y Moreno Mena, J. C. (2009). Buenas Prácticas Agrícolas en el Cultivo de Plátano. En J. M. Men, Reducción del Escurrimiento de Plaguicidas al Mar Caribe (págs. 10-40). Medellín: Impresos S.A.

Ministerio de Agricultura. (1994). Notas de química de suelos. La Habana, Cuba: Instituto de Suelos.

Montoya María L. R., M. F. (Mayo de 2014). Impacto del manejo de agroquímicos, parte alta de la microcuenca Chorro Hondo. Recuperado el 2 de Octubre de 2016, de Revista facultad nacional de salud pública: http:// wWw.scielo.org.co/scielo.php?script=sci_arttext\&pi$d=$ S0120-386X2014000200004

Narro, E. (1994). Física de suelos con enfoque agrícola. México: Trillas. pp. 50

Noa-Carrazana, J.C. y Flores-Estévez, N. (2008). Los plátanos en el ecosistema de montaña y su relación con el virus del rayado del plátano (BSV). En:. Sánchez-Velázquez, L.R., Díaz-Fleischer, F., Galindo-González, J. (eds.), Ecología, Manejo y Conservación de los Ecosistemas de Montañas en México. Ediciones CONABIO, Editorial MUNDI PRENSA, DF, México. pp. 379-393.

Organización Mundial de la Salud. (2016). Inocuidad de 
los alimentos. Recuperado el 14 de Septiembre de 2016, de Organización mundial de la salud: http://www.who.int/ topics/food_safety/es/

Pereira, C., Mauro, F., Maycotte, C., Restrepo, B., Calle, A., y Esther, M. (2011). Sistema de producción vegetal II. Colombia: Espacio Gráfico Comunicaciones S.A.

Porta Jaime, López Marta \& Roquero Carlos. (1999). Degradación de suelos en relación con prácticas agrícolas. En Edafología para la agricultura y el medio ambiente (775-779). Madrid: Mundi-Prensa.

Secretaría de Agricultura Ganadería Desarrollo Rural Pesca y Alimentos. (2005). Plan rector sistema producto nacional plátano. México: SAGARPA.

Secretaría de Agricultura Ganaderia Desarrollo Rural Pesca y Alimentos. (2012). Plan de manejo y recolección de envases vacíos de agroquímicos. México: SAGARPA.

Sparks, D. (1995). Environmental soil chemistry. Academic Press.

Uzcátegui, J., Araujo, Y., Mendoza, L. (2011). Residuos de plaguicidas organoclorados y su relación con parámetros físico-quimicos en suelos del municipio pueblo llano. Mérida: Universidad de Los Andes.

Castro T.F. y Yoshida T. 1971. Degradation of organochlorine insecticides in flooded soils in the Philippines. J. Agr. Food Chem. 19: 1168-1170. 